tions. For the purpose of evaluating what states or conditions favor those seizures, the author investigated histologically the process of healing of the operative wound in cats' brains and compared the postoperative threshould of convulsive seizures with the preoperative one by intravenous metrazol injection.

The following results were obtained.

The width and density of the meningeal adhesion in part of the cerebral wound were well correlated with the decrease of the threshold of convulsive seizures.

Histological observation of the process of healing mechanism in the cerebral wound revealed that mixed cleaning and organization by mesenchymal as well as glial cells occur at the site of injury (wound) and the mesenchymal reaction is more marked in the cortex, while the glial one is more in the white matter.

Meningeal adhesion does not always occur in the cerebral wound. The deeper and greater the wound is, the less the incidences of adhesion. Both the mesenchymal and the glial reaction are more marked in cases of healing with adhesion than in those without adhesion.

\title{
11. A Case of KLIPPEL-FEIL Syndrome
}

\author{
K. Kitamura \\ Tokai Chuo Hospital
} \begin{abstract}
of legs.
A man aged 44, complained of unsteadiness in walking and paresthesia
\end{abstract}

At the age of 35 , he experienced paresthesia and pair in the rigit shoulder, and 5 years apart, he has had paresthesia of the lower extremity and difficulty in walking and the left scapular and deltoidal muscle atrophy began. Since that time, these symptoms gradually progressed.

In general appearance, the neck was very short and its movement were disturbed. The left upper extremity was atrophic and the left hand grasping power was very poor. Examination of the cranial nerves revealed no significant changes. There was no involuntary movement. Bilaterally, Hoffmann's, Rossolimo's, and Puusepp's sign was present, abdominal reflexes disappeared, P.T.R. and A.T.R. was overactive and ankle clonus was present. There were marked diminished protopathic, two-point discremination and vibratory sense below the $\mathrm{L}_{1}$ spinal dermatomes and slightly diminished kinetic and postural sense.

Röntgenogram of cervical spine revealed, that there was fusion of the bodies of the I, II and III and of the V, VI and VII cervical vertebrae and the number of spinous process was only four. 